\title{
Neuritis óptica atípica en un adulto joven: un desafío diagnóstico
}

\section{Atypical optic neuritis in a young adult: a diagnostic challenge}

Marcela Triviño I. ${ }^{1}$, Federica Solanes ${ }^{2}$ y Cristian Salgado A. ${ }^{3}$

Optic neuritis is an inflammatory disease of the optic nerve and is classified into typical and atypical: the first, may be associated with diagnostic or future risk of developing multiple sclerosis (MS). However, the differential diagnosis is extensive and prognosis and treatment depend on the cause. The purpose of this paper is to present a case of demyelinating optic neuritis and discuss the diagnosis step confrontation.

Key words: Optic nerve diseases, optic neuritis, neuromyelitis optica.

Rev Chil Neuro-Psiquiat 2014; 52 (2): 81-88

\section{Introducción}

$\mathrm{L}$ a neuritis óptica es una enfermedad inflamatoria aguda del nervio óptico ${ }^{1-3}$. La podemos clasificar de varias formas, siendo las más frecuentes según el patrón clínico (típica $v s$ atípica) o según la causa subyacente: EM, neuromielitis óptica (NMO), asociada a enfermedades inmunológicas e infecciosas sistémicas, e idiopática. Las formas típicas se pueden asociar a EM al momento del diagnóstico o interpretarse como un síndrome desmielinizante aislado con riesgo de conversión a EM en el futuro. Las formas atípicas pueden clasificarse en aquellas asociadas o no a enfermedades sistémicas. Dentro de las primeras destacan las enfermedades del tejido conectivo, vasculitis, infecciones y sarcoidosis. Dentro de las segundas se incluyen la NMO y la neuritis óptica crónicarecurrente idiopática dependiente de corticoides ${ }^{1-5}$.
El diagnóstico de neuritis óptica es eminentemente clínico, siendo los exámenes complementarios de ayuda en el diagnóstico diferencial ${ }^{1-3}$. La RM evidencia la lesión inflamatoria del nervio óptico en el 95\% de los casos, permite realizar el diagnóstico o estratificar el riesgo de conversión a EM, puede mostrar otras causas de neuritis óptica y mostrar el grado de compromiso ${ }^{3}$. Los exámenes inmunológicos e infecciosos permiten identificar causas secundarias de neuritis óptica, principalmente en los casos atípicos ${ }^{1-5}$. La tomografía de coherencia óptica (OCT: optical coherence tomography) mide el grosor de la capa de fibras nerviosas (CFN) de la papila; en forma temprana puede mostrar aumento del grosor por edema, en forma más tardía el grosor será menor por pérdida de neuronas a causa del episodio de neuritis. En el caso de EM el compromiso es de predominio temporal y en NMO de los polos superior e inferior ${ }^{7,8}$.

Recibido: 09/12/2013

Aceptado: 16/06/2014

Los autores no presentan ningún tipo de conflicto de interés.

Residente de Oftalmología, Pontificia Universidad Católica de Chile. Santiago, Chile.

2 Médico Oftalmólogo, Pontificia Universidad Católica de Chile. Santiago, Chile.

3 Médico Oftalmólogo, Profesor Asistente, Pontificia Universidad Católica de Chile. Santiago, Chile. 
Los potenciales visuales evocados (PVE) en general no son necesarios, a menos que el diagnóstico de neuritis óptica desmielinizante (NOD) sea dudoso, mostrando aumento del tiempo de conducción?.

Otras causas de neuropatía óptica (isquémica, traumática, compresiva, hereditaria, tóxico-metabólica, congénitas, etc.) las diferenciamos por el examen oftalmológico, la alteración de campo visual, la evolución natural y los exámenes complementarios (neuroimágenes y laboratorio), pero el detalle es tan extenso que queda fuera de esta publicación.

El propósito del presente trabajo es presentar un caso clínico sobre NOD, y cómo un cuadro atípico se convierte en un desafío diagnóstico que nos exige ampliar nuestras hipótesis, para asegurar a nuestro paciente un diagnóstico certero y tratamiento adecuado.

\section{Caso clínico}

Paciente de sexo masculino de 37 años, con antecedente de tabaquismo y depresión en tratamiento. Consulta por cuadro de 2 semanas de evolución caracterizado por pérdida de la AV del ojo izquierdo (OI), tipo escotoma central, con progresión en los días siguientes, asociada a cefalea frontal leve, sin exacerbación con los movimientos oculares. Refiere además parestesias en hemicuerpo izquierdo. Historia familiar negativa para enfermedades oculares y neurológicas.

$\mathrm{Al}$ examen oftalmológico destaca: agudeza visual mejor corregida (AVMC) ojo derecho (OD) 1,0 y en OI cuenta dedos a 1 metro, visión de colores (test de Ishihara) OD 14/14 y OI 0/14, pupilas isocóricas con reflejo fotomotor (RFM) directo enlentecido en ambos ojos (AO) OD +2 y OI +1,5 y defecto pupilar aferente relativo (DPAR) positivo en OI, alineado en posición primaria de mirada, oculomotilidad completa normal y no dolorosa, movimientos sacádicos y de seguimiento normales, sin alteraciones al examen de los nervios craneanos (NC) V, VII y VIII, biomicroscopía normal en AO, papilas rosadas de bordes netos, excavación $0,3 \mathrm{AO}$ con buen reborde neurorretinal, máculas $\mathrm{AO}$ sin lesiones.
Se solicita campo visual computarizado Humphrey 30-2 sita standard (CV 30-2 SS) que muestra: OD hemianopsia temporal incompleta (defecto medio (DM) -4,22 decibeles ( $\mathrm{dB})$ ), y OI defecto centrocecal (DM -4,50 dB) (Figura 1A). Con la sospecha de síndrome quiasmático de la rodilla izquierda se solicita resonancia nuclear magnética (RNM) de cerebro con y sin gadolinio que evidencia múltiples lesiones hiperintensas en sustancia blanca supra e infratentorial en secuencias FLAIR/ T2, de distribución predominantemente periventricular supratentorial, asociado a compromiso del nervio óptico izquierdo prequiasmático con compromiso del margen quiasmático, todas lesiones compatibles con enfermedad desmielinizante (Figura 2A, 2B y 2D), y refuerzo con contraste endovenoso de algunas lesiones subcorticales frontales izquierdas, otra periventricular ipsilateral y la lesión del nervio óptico izquierdo (Figura $2 \mathrm{C} \mathrm{y}$ 2E). Posteriormente, se solicita RNM de columna total que muestra una lesión medular, hiperintensa en T2 a la altura de C1-C2 de $8 \mathrm{~mm}$ de diámetro mayor, de localización posterior y lateralizada a derecha, que no se refuerza con medio de contraste, compatible con lesión desmielinizante (Figura 2F). En la evaluación por neurología no se detectaron otros hallazgos.

El LCR no presentaba células, albúmina LCR 40,4 (VN hasta 35), IgG 5,1 mg/dL (VN hasta $3,4 \mathrm{mg} / \mathrm{dL})$, sin aumento del índice de $\operatorname{IgG}$ (IgG LCR/IgG suero $0,51, \mathrm{VN}$ : hasta 0,7$)$, y sin bandas oligoclonales (BOC). Se complementa estudio con exámenes de laboratorio general destacando hemograma normal, VHS $3 \mathrm{~mm} / \mathrm{h}$, perfil bioquímico normal, perfil reumatológico con anticuerpos antinucleares (ANA) positivo $1 / 320$ con patrón homogéneo y anticuerpo anti proteína p-ribosomal dentro de límites normales, perfil ENA negativo, y estudio infeccioso con VDRL y VIH ambos negativos.

En el contexto de síndrome quiasmático anterior, con lesiones desmielinizantes múltiples en el sistema nervioso central (SNC) tanto supra como infratentoriales que cumplen los criterios de McDonald, la ausencia de criterios NMO en RNM y LCR, y la falta de evidencia para afirmar una enfer- 


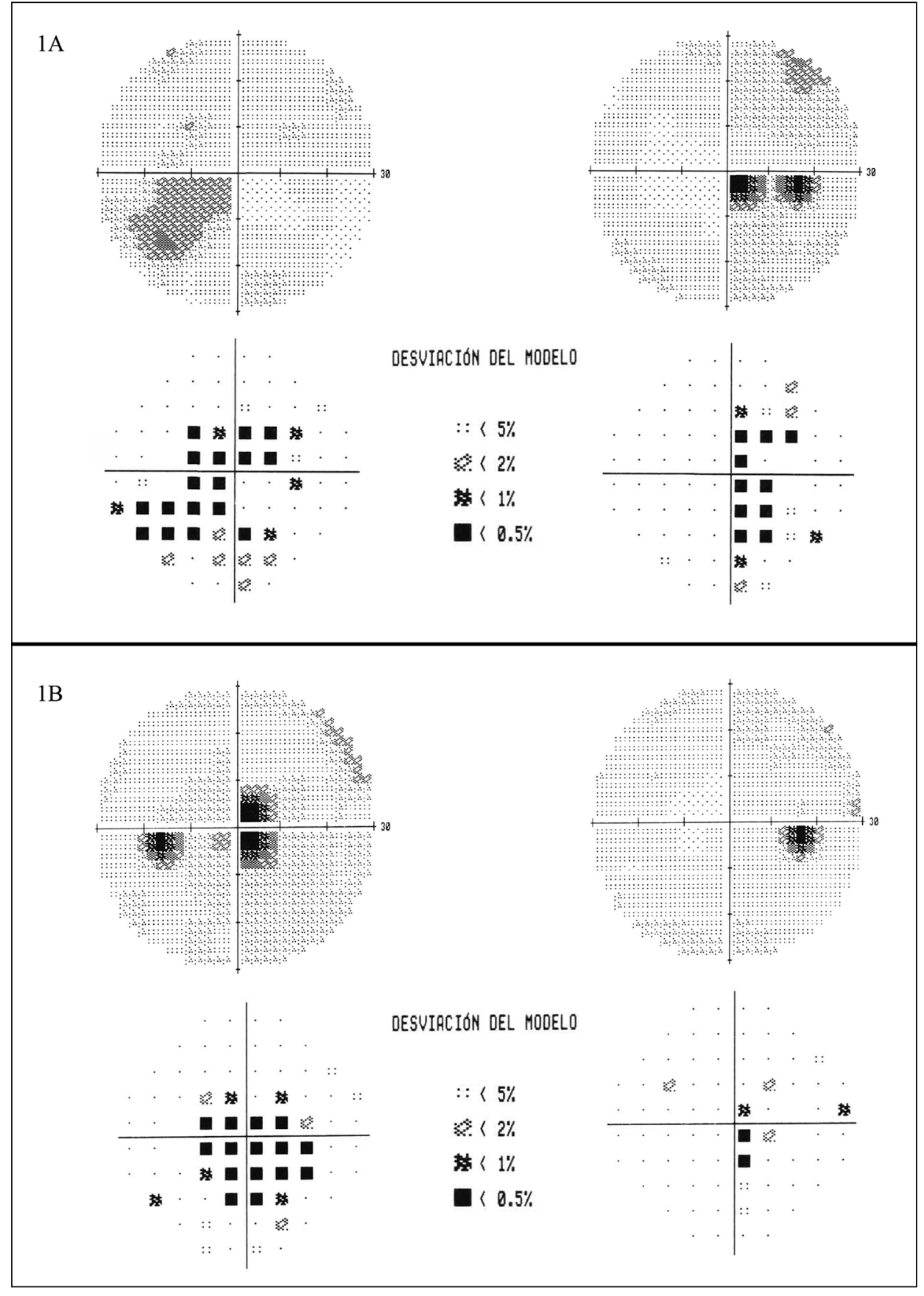

Figura 1. Campos visuales computarizados Humphrey $30-2$, en cada figura: a la izquierda OI, a la derecha OD, arriba escala de grises, abajo rejilla de desviación modelo. A) Campo visual basal: se observa una hemianopsia temporal incompleta en OD (DM -4,22 dB) y un defecto centrocecal en OI (DM -4,50 dB). B) Campo visual de control: se observa un escotoma central mínimo en $\mathrm{OD}(\mathrm{DM}-3,47 \mathrm{~dB})$ y un escotoma centrocecal en OI de menor extensión que en el examen basal (DM $-7,48 \mathrm{~dB})$. medad inmunológica, se diagnostica primer brote de esclerosis múltiple. No se indican corticoides para el manejo de la neuritis, dado que el paciente no cumple con los criterios de temporalidad del estudio Optic Neuritis Treatment Trial (ONTT) para hacerlo.
En control clínico a las 6 semanas de iniciado el cuadro, el paciente evoluciona con mejoría leve de la AVMC $(\mathrm{OI} 0,15)$ y de la visión de colores (OI 2,5/10), persistiendo con DPAR positivo OI con filtro 0,3 , y CV 30-2 SS OD escotoma central mínimo (DM -3,47 dB) y OI escotoma centro- 


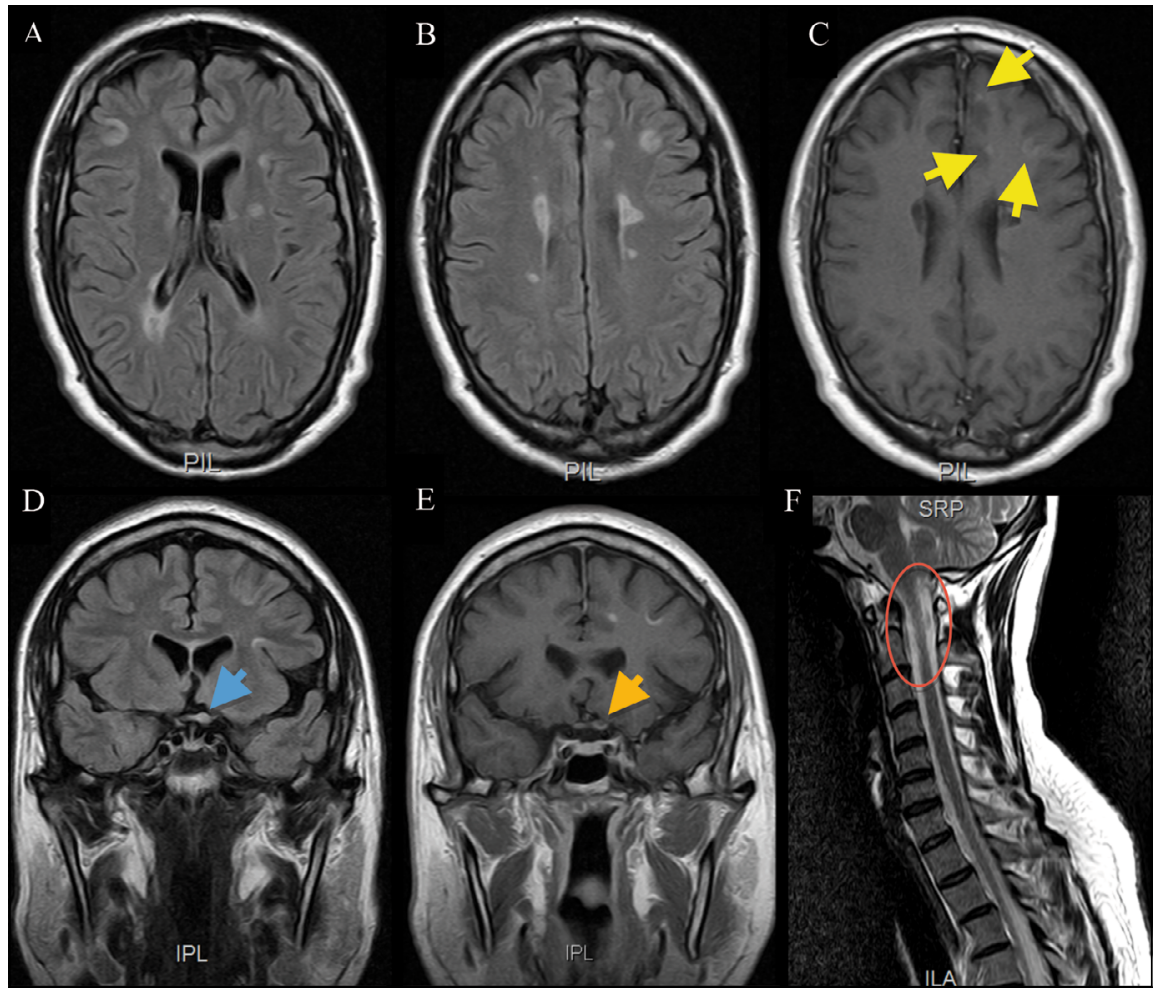

Figura 2. A y B) RNM de cerebro en T2/FLAIR: lesiones hiperintensas periventriculares y subcorticales (lesiones blanquecinas). C) RNM de cerebro con gadolinio: captación de contraste por lesiones frontales y periventricular (flechas amarillas). D) RNM de cerebro en T2/FLAIR: lesión hiperintensa quiasmática anterior izquierda (flecha celeste). E) RNM de cerebro con gadolinio: captación de contraste por nervio óptico prequiasmático izquierdo (flecha naranja). F) RNM de columna en T2: lesión hiperintensa entre $\mathrm{C} 1$ y C2 (óvalo rojo). cecal de menor extensión que en examen previo (DM -7,48 dB) (Figura 1B). El OCT de papilas muestra en OI disminución del grosor de CFN en cuadrante temporal, sin alteraciones en OD. A los 5 meses, el paciente ya presenta mejoría significativa de AVMC (OI 0,6), de la visión de colores (OI 10/10), del DPAR (positivo sólo con filtro 0,6 ) y de los defectos campimétricos (OI DM -5,75 dB con escotoma centrocecal menor que el previo). A los 12 meses destaca recuperación completa de la AV (OI 1,0) y del DPAR (negativo), con papilas de aspecto normal sin evidencia clínica de atrofia óptica en AO.

\section{Discusión}

La neuritis óptica típica se caracteriza por una disminución monocular aguda de la visión, dolorosa con los movimientos oculares en el 90\% de los pacientes, que puede progresar durante la primera semana y que se recupera espontáneamente entre las 2 a 4 semanas de iniciado el cuadro ${ }^{10}$. Existe una alteración variable de la AVMC, los reflejos pupilares y la percepción de colores. El examen de los nervios ópticos en 2/3 de los casos es normal (debido a que el compromiso es retrobulbar); el hallazgo de palidez papilar indica antigüedad de al menos 5-6 semanas (en los casos en los que se produce atrofia) y a veces puede encontrarse uveítis intermedia $^{1-5}$. En el campo visual pueden encontrarse varios tipos de defectos: difusos (48\%), localizados (20\%, incluye altitudinales o arqueados), centrales o centrocecales $(8 \%)$, otros $(23 \%)^{11}$. El uso de metilprednisolona endovenosa por 3 días seguido de prednisona por 11 días, indicados hasta 3 días del inicio de los síntomas, acelera el tiempo de recuperación, pero no afecta el pronóstico visual final ${ }^{12,13}$.

Las formas típicas de NOD son predominantemente idiopáticas, pero se pueden asociar a EM al momento del diagnóstico o interpretarse como un síndrome desmielinizante aislado con riesgo de conversión a $\mathrm{EM}^{1-5}$. La NOD aguda puede ser la primera manifestación de EM en el 15 a 20\% de 
las personas con esta enfermedad, o puede ocurrir posteriormente durante el curso de la enfermedad hasta en el 50-70\% de ellas ${ }^{4-9}$. De acuerdo al ONTT, la probabilidad de desarrollar EM posterior a un episodio de NOD unilateral en pacientes con RNM de cerebro basal normal, comparados con aquellos con 1 o más lesiones desmielinizantes, fue $16 \%$ vs $51 \%$ a 5 años, $22 \%$ vs $56 \%$ a 10 años, y $25 \%$ vs $72 \%$ a 15 años, respectivamente ${ }^{14}$. Según los criterios de McDonald, el diagnóstico de EM requiere la presencia de lesiones diseminadas en espacio (al menos una lesión en T2 en al menos 2 de los siguientes 4 sitios: yuxtacortical, periventricular, infratentorial y médula espinal) y tiempo (evidencia de nueva lesión en T2 en una RNM de control o nueva captación de gadolinio de una lesión antigua, o presencia simultánea de lesiones asintomáticas captantes y no captantes de gadolinio, en cualquier momento ${ }^{15}$. Respecto a las lesiones encontradas en la RNM de columna, en la EM la mielitis producida es pequeña, asimétrica y focal, de localización frecuentemente dorsal, y las lesiones rara vez exceden 1 ó 2 segmentos vertebrales ${ }^{16-18}$. La punción lumbar (PL) puede presentar aumento de inmunoglobulinas, y en el $80 \%$ de los pacientes presenta bandas oligoclonales $(\mathrm{BOC}+)$, pero estas alteraciones no son específicas de EM. En la EM, el compromiso de la CFN en el OCT tiende a ser de predominio temporal y puede demostrar alteraciones subclínicas ${ }^{1-5}$.

Las neuritis ópticas atípicas se caracterizan por compromiso bilateral o secuencial de ambos nervios ópticos, patrón de pérdida visual más severo o prolongado, ausencia de recuperación, alteraciones en el fondo de ojos diferentes a las descritas previamente y síntomas y signos de enfermedades sistémicas.

La NMO es una enfermedad autoinmune desmielinizante del SNC que afecta principalmente la médula espinal y los nervios ópticos ${ }^{5}$. Existe una sobreposición de síntomas entre EM y NMO, pero estas patologías corresponden a entidades con características fisiopatológicas, clínicas, radiológicas diferentes y evolutivas. Las características cardinales de NMO son la presencia de mielitis transversa, que se observa como lesiones hiperintensas cen- trales en T2 con compromiso longitudinal de 3 o más segmentos vertebrales contiguos (a diferencia de EM en que el compromiso es menos extenso), $y$ neuritis óptica frecuentemente bilateral (simultánea o secuencial) $)^{5,6}$. Estos eventos de mielitis y neuritis en NMO pueden ocurrir simultáneamente, en sucesión rápida, o separados por varios años ${ }^{5,18,19}$. Respecto a las neuroimágenes, en NMO la RNM de cerebro es típicamente normal, excepto por la captación de gadolinio del nervio óptico durante un ataque agudo de neuritis óptica; sin embargo, pueden presentar lesiones en la materia blanca que son inespecíficas y que, por definición, no cumplen criterios para el diagnóstico de EM. Las lesiones cerebrales aparecen en el $60 \%$ de los pacientes más tarde en el curso de la enfermedad, pero estas lesiones generalmente son asintomáticas. Adicionalmente, pueden presentar lesiones de tronco que pueden ocurrir en forma aislada o como extensión rostral (cefálica) de una mielitis cervical ${ }^{16-18}$. El estudio de RNM de columna es característico en $\mathrm{NMO}$, evidenciando una mielitis con compromiso de 3 o más segmentos consecutivos, de localización predominantemente central, con expansión medular en fase aguda, lesión hipointensa en T1 e hiperintensa en T2, con captación de gadolinio variable $^{16-18}$. La PL puede mostrar pleocitosis y ausencia de BOC en el 70-80\% ${ }^{17,18}$. El año 2004 se descubrió un autoanticuerpo marcador de NMO, NMO-IgG, dirigido contra el canal de agua aquaporina -4 con alta sensibilidad (68-91\%) y especificidad (8599\%) para $\mathrm{NMO}^{20}$. Finalmente, el pronóstico y la respuesta a tratamiento de los pacientes con NMO es peor que en los casos de $\mathrm{EM}^{5}$.

Algunas enfermedades autoinmunes sistémicas, tales como lupus, síndrome de Sjogren, esclerosis sistémica, enfermedad de Behçet y síndrome antifosfolípidos pueden asociarse a lesiones desmielinizantes del sistema nervioso central. En estos pacientes las características de las alteraciones en la RM, la clínica y el estudio sistémico en fundamental para un adecuado diagnóstico y tratamiento, dado que los síntomas y signos se sobreponen con los de $\mathrm{EM}$ y $\mathrm{NMO}^{21}$.

La encefalomielitis aguda diseminada (ADEM: acute difusseminated encephalomyelitis) es una 
enfermedad que se relaciona con el uso de vacunas e infecciones en $50-75 \%$ de los casos hasta 4 semanas antes del debut, que afecta principalmente a niños y que provoca compromiso difuso del sistema nervioso central con encefalopatía y múltiples manifestaciones neurológicas simultáneas, dentro de las cuales está la neuritis óptica. En la RNM se observan lesiones diseminadas, multifocales y extensas, que afectan tanto sustancia blanca como gris y que desaparecen o permanecen estables en el tiempo. En la PL puede haber pleocitosis linfocítica, aumento de albúmina y BOC+ transitorias hasta el 12\% de los casos. En general la enfermedad es monofásica y de buen pronóstico, pero hasta un tercio de los pacientes pueden presentar recaídas dentro de 3 meses del primer episodio ${ }^{22}$.

En el caso clínico presentado, el paciente presentó síntomas y signos compatibles con NOD pero con características que la clasifican como atípica (ausencia de dolor ocular espontáneo y con los movimientos oculares, sin recuperabilidad significativa de la AVMC aún a las 4 semanas y compromiso bilateral clínico y en el CV por compromiso quiasmático), lo que nos llevó a ampliar el diagnóstico diferencial. Del estudio sistémico, no hubo evidencia clínica ni de laboratorio para confirmar otras causas de neuritis óptica, como las infecciosas y autoinmunes. El estudio de neuroimágenes fue clave para definir la causa, ya que la RNM de cerebro confirmó el compromiso de la rodilla quiasmática anterior izquierda concordante con el CV, y mostró múltiples lesiones que cumplen con los criterios de McDonald de diseminación en espacio y tiempo para el diagnóstico de EM. Sin embargo, sabemos que el compromiso quiasmático es más frecuente en NMO que en EM, y la presencia de lesiones típicas de EM en RNM de cerebro no descartan el diagnóstico de NMO si se demuestra que cumple criterios para esta última ${ }^{18}$. De esta manera, el estudio de RNM de columna total no mostró lesiones con características propias de NMO, sino más bien de EM. Adicionalmente, el estudio del LCR mostró un aumento de la IgG, alteración que ha sido descrita como más característica de EM, pese a la ausencia de BOC positivas (15\% de personas con EM no tienen BOC en el LCR $)^{24}$.

Por otra parte, el estudio de PVE en nuestro paciente resultó en compromiso desmielinizante bilateral, concordante con compromiso quiasmático anterior, aunque sabemos que que los PVE no tienen un valor discriminatorio entre EM o NMO, sino más bien permiten aproximarse cuando el diagnóstico de NOD es dudoso ${ }^{25}$. El OCT arrojó una disminución del grosor de CFN en cuadrante temporal del OI, lo que se corresponde con el escotoma centrocecal en OI debido al compromiso de las fibras del haz máculo-papilar, y sin compromiso de la CFN en OD, todo compatible con el compromiso quiasmático anterior y la resolución significativa del defecto de CV en OD.

Dado el diagnóstico final de primer brote de esclerosis múltiple, el paciente comenzó tratamiento con interferón beta 1-a semanal.

\section{Conclusiones}

$\mathrm{Al}$ enfrentar un paciente con daño funcional del nervio óptico, debemos considerar las diferentes causas de neuropatía óptica, dentro de las cuales una posibilidad es la neuritis óptica. Es importante recordar que no toda NOD se asocia a EM, sino que debemos estar alerta a aquellos cuadros clínicos que no son característicos de EM, y solicitar los exámenes necesarios para descartar o confirmar otras etiologías posibles. Lograr un diagnóstico certero en pacientes con neuritis óptica atípica nos permitirá iniciar un tratamiento adecuado, logrando a largo plazo mejores resultados funcionales que permitan preservar una mejor calidad de vida en nuestros pacientes. 


\section{Resumen}

La neuritis óptica es una enfermedad inflamatoria del nervio óptico y se clasifica en típica y atípica: la primera, se puede asociar al diagnóstico o riesgo futuro de desarrollo de esclerosis múltiple (EM). Sin embargo, el diagnóstico diferencial es extenso y el tratamiento y pronóstico dependen de la causa. El propósito del presente trabajo es presentar un caso clínico sobre neuritis óptica desmielinizante y discutir paso a paso el enfrentamiento diagnóstico.

Palabras clave: Neuropatía óptica, neuritis óptica, neuromielitis óptica.

\section{Referencias bibliográficas}

1. Balcer LJ. Clinical practice. Optic neuritis. N Engl J Med 2006; 354 (12): 1273-80.

2. Hoorbakht H, Bagherkashi F. Optic Neuritis, its Differential Diagnosis and Management. Open Ophthalmol J 2012; 6: 65-72.

3. Toosy, Mason, Miller. Optic neuritis. Lancet Neurol 2014; 13: 83-99.

4. Frohman, Frohman, Zee, McColl, Galetta. The neurophtahlmology of multiple Sclerosis. Lancet Neurol 2005; 4: 111-21.

5. Drori, Cahpman. Diagnosis and classification por neuromylitis óptica (Devic's Syndrome). Autoimmunity reviews 2014; 531-3.

6. Petzold, Plant. Diagnosis and classification of autoinmune óptica neuropathy. Autoimmunity reviews 2014: 539-45.

7. Kallenbach K, Frederiksen J. Optical coherence tomography in optic neuritis and multiple sclerosis: a review. Eur J Neurol 2007; 14 (8): 841-9.

8. Vidal-Jordana Á, Sastre-Garriga J, Montalban X. Optical coherence tomography in multiple sclerosis. Rev Neurol 2012; 54 (9): 556-63.

9. Kolappan M, Henderson AP, Jenkins TM, Wheeler-Kingshott CA, Plant GT, Thompson AJ, et al. Assessing structure and function of the afferent visual pathway in multiple sclerosis and associated optic neuritis. J Neurol 2009; 256 (3): 305-19.

10. Beck RW, Cleary PA, Backlund JC. The course of visual recovery after optic neuritis. Experience of the Optic Neuritis Treatment Trial. Ophthalmology 1994; 101: 1771-8.

11. Optic Neuritis Study Group. The clinical profile of optic neuritis. Experience of the Optic Neuritis
Treatment Trial. Arch Ophthalmol 1991; 109: 1673-8

12. Gal RL, Vedula SS, Beck R. Corticosteroids for treating optic neuritis. Cochrane Database Syst Rev 2012; 4 .

13. Brodsky M, Nazarian S, Orengo-Nania S, Hutton GJ, Buckley EG, Massey EW, et al. Optic Neuritis Study Group. Multiple sclerosis risk after optic neuritis: final optic neuritis treatment trial followup. Arch Neurol 2008; 65 (6): 727-32.

14. The Optic Neuritis Study Group. Multiple Sclerosis Risk After Optic Neuritis: Final Optic Neuritis Treatment Trail Follow-Up. Arch Neurol 2008; 65 (6): 727-32.

15. Polman CH, Reingold SC, Banwell B, Clanet M, Cohen JA, Filippi M, et al. Diagnostic criteria for multiple sclerosis: 2010 revisions to the McDonald criteria. Ann Neurol 2011; 69 (2): 292-302.

16. Wingerchuk DM, Lennon VA, Lucchinetti CF, Pittock SJ, Weinshenker BG. The spectrum of neuromyelitis optica. Lancet Neurol 2007; 6 (9): 805-15.

17. Anu Jacob, Mike Boggild. Neuromyelitis óptica. Practical Neurology 2006; 6: 180-4.

18. Wingerchuk DM. Diagnosis and treatment of neuromyelitis óptica. Neurologist 2007; 13 (1): 2-11.

19. Jacob, Mike Boggild. Neuromyelitis óptica. Practical Neurology 2006; 6: 180-4.

20. Lennon VA, Wingerchuk DM, Kryzer TJ, Pittock SJ, Lucchinetti CF, Fujihara K, et al. A serum autoantibody marker of neuromyelitis optica: distinction from multiple sclerosis. Lancet 2004; 364 (9451): 2106-12.

21. Cikes N, Bosnic D, Sentic M. Non-MS autoimmu- 
ne demyelination. Clin Neurol Neurosurg 2008; 110 (9): 905-12.

22. Karussis D, Petrou P. The spectrum of postvaccination inflammatory CNS demyelinating syndromes. Autoimmun Rev 2014; 13 (3): 215-24.

23. Menge T, Hemmer B, Nessler S, Wiendl H, Neuhaus $\mathrm{O}$, Hartung HP, et al. Acute disseminated encephalomyelitis: an update. Arch Neurol 2005; 62 (11): 1673-80.
24. Carroll W, Saida T, Kim H, Kira J, Kermode A, Tsai C, et al. A guide to facilitate the early treatment of patients with idiopathic demyelinating disease (multiple sclerosis and neuromyelitis optica). Mult Scler 2013; 19 (10): 1371-80.

25. Schäffler N, Köpke S, Winkler L, Schippling S, Inglese $\mathrm{M}$, Fischer $\mathrm{K}$, et al. Accuracy of diagnostic tests in multiple sclerosis-a systematic review. Acta Neurol Scand 2011; 124 (3): 151-64.

\section{Correspondencia:}

\section{Cristian Salgado Alarcón}

Clínica UC San Carlos de Apoquindo.

Camino el Alba 12351. Las Condes.

Teléfono: (022) 7548934. Celular: 62361998.

E-mail: csalgado@med.puc.cl 